\title{
Anabases
}

ANABASES Traditions et réceptions de l'Antiquité

$24 \mid 2016$

Varia

\section{Le Cardinal Capella de Henry de Montherlant. Fragments d'un récit inachevé}

Pierre Duroisin

\section{(2) OpenEdition}

1 Journals

Édition électronique

URL : http://journals.openedition.org/anabases/5959

DOI : 10.4000/anabases.5959

ISSN : 2256-9421

Éditeur

E.R.A.S.M.E.

Édition imprimée

Date de publication : 10 novembre 2016

Pagination : 183-200

ISSN : 1774-4296

\section{Référence électronique}

Pierre Duroisin, « Le Cardinal Capella de Henry de Montherlant. Fragments d'un récit inachevé », Anabases [En ligne], 24 | 2016, mis en ligne le 01 octobre 2019, consulté le 22 mars 2020. URL : http:// journals.openedition.org/anabases/5959; DOI : https://doi.org/10.4000/anabases.5959 


\title{
Le Cardinal Capella de Henry de Montherlant Fragments $d$ 'un récit inachevé
}

Pierre Duroisin

\begin{abstract}
E n 2013, Anabases a publié un manuscrit de Montherlant conservé à la - Bibliothèque nationale de France qu'il avait paru légitime d'intituler Jules César'. Le manuscrit qu'on présente aujourd'hui sous un titre qui va pareillement de soi : Le Cardinal Capella, appartient au même fonds.

Le document compte vingt feuillets, un feuillet de notes, réparties sur les deux faces d'une lettre des Éditions du Capitole datée du 27 février $1930^{2}$, pour dix-neuf feuillets d'un texte à lire en trois temps : onze pages numérotées, par l'auteur, de 1 à 11 , quatre pages numérotées de 1 à 4 et de nouveau quatre pages numérotées de 1 à 4, chaque section correspondant à une étape du récit. Récit hélas incomplet sans qu'on puisse dire si Montherlant s'arrêta tout net dans son effort ou si les parties manquantes se sont volatilisées. Il ne semble pas, en tout cas, que l'écrivain ait jamais fait, dans ses carnets ou ailleurs, la moindre allusion à Capella.
\end{abstract}

1 Voir “ Le Jules César de Montherlant : dialogue avec une ombre » dans Anabases, 18 (2013), p. 131-174. Comme pour Jules César, c'est grâce à M. Jean-Claude Barat, l'ayant droit de Montherlant, que j'ai pu lire Le Cardinal Capella dans les meilleures conditions. Je veux aussi dire ma reconnaissance à M. Guillaume Fau, responsable du fonds Montherlant au département Manuscrits de la $\mathrm{BnF}$, qui a répondu à tous mes appels avec un rare empressement, au service de reproductions de la BnF pour la qualité de son travail et, plus encore, à la revue Anabases pour l'accueil qu'elle réserve à mes travaux sur Montherlant.

2 La lettre, adressée à « Monsieur H. de Montherland (sic) 41 rue de Bourgogne », reprend les deux versements, de 4000 et de 3000 frs, qui lui ont été faits en 1929 pour Sous les drapeaux morts. La date n'est pas d'une clarté absolue, le 2 dactylographié du 27 surchargeant on ne sait trop quoi, mais il s'agit à coup sûr d'une date à deux chiffres. 
De quand date ce projet abandonné ? La réponse est a priori donnée par la lettre des Éditions du Capitole, mais nous reviendrons sur ce point après avoir lu le texte. Retenons seulement, pour l'instant, le mot “ CAPELLA » qui figure en grandes capitales et au crayon gras au beau milieu de la première page de notes ${ }^{3}$.

\section{Le texte}

Le cardinal Capella tenait la tête baissée sur un plan de Rome et, dans un cercle de traits de plume, délimité par les sept collines, son esprit tournait comme dans une cage. Le nom de chaque quartier de la ville lui rappelait quelque chose d'infiniment particulier. Partout, partout, il y avait la demande de ce qu'il cherchait. Et la ville, étalée sur le papier, pas plus grande qu'une main, lui montait à la tête.

Il releva la tête, la gravité, ou plutôt l'expression immobile et fermée de son visage l'effraya. Tout ce qu'il y avait d'accusateur dans ce visage! - dans cette bouche pincée, qui peu à peu s'était pincée pour garder un secret dans les poches mauves sous ses yeux. On l'a dit, mais on ne le dira jamais assez : le seul secret pour garder longtemps le rose des joues et la jeunesse du sourire est de paraître ce qu'on est, de ne pas faire l'effort d'être deux. Les visages usés sont ceux des importants et des hypocrites. Le cardinal Capella était l'un et l'autre.

L'appréhension de ce qu'il ferait cette nuit tirait son visage, l'empêchait complètement de porter son attention sur quoi que ce soit d'autre. Ardemment, il souhaitait que Perotto lui apportât un non.

Sur la table, en désordre, était un monceau d'actes. Son regard tomba sur eux et il soupira. La nécessité de mener de front les affaires et les plaisirs l'épuisait. Encore, les affaires traînaient-elles depuis une semaine, il y avait là des dossiers urgents qui attendaient.

- Il ne viendra pas, se disait le cardinal. Peut-être qu'il n'a pas besoin d'argent en ce moment. Le diable emporte les gens qui par moments sont désintéressés ! On ne peut jamais compter sur eux. Il va inventer n'importe quelle histoire pour m’expliquer que sa démarche a échoué.

On frappa à la porte du vaste cabinet, et son cœur battit. Un jeune homme, de dix-sept ans environ, entra.

- Eh bien ? dit le cardinal.

- On vous attend cette nuit, à onze heures.

Quand, il y a une semaine, Perotto avait annoncé à son maître : " C'est une affaire arrangée ", à l'instant, le cardinal s'était mis à trembler. Il tremblait, de bouleversement, tandis que Perotto lui expliquait la chose, comme lorsque, en

3 Le document, que la BnF a sobrement intitulé Capella, n'est pas encore folioté. Il porte les références « NAF 28165, boîte 16 (Varia) : Textes des années 1929-1930 ». 
décembre, on ouvre brusquement les vitres d'une pièce. Enfin, il avait mordu son mouchoir, de joie.

Un abîme s'ouvrait devant le cardinal. Cela dut se voir à l'expression de son visage, car le jeune homme dit :

- On dirait que vous n'êtes pas content. Moi qui attendais des éloges!

- Mon pauvre Perotto, tu sais comme je suis, dit le cardinal, avec une lassitude de lui-même qui se sentit dans sa voix. Toujours à aimer ce que je n'ai pas, et à ne plus aimer ce que j'ai. Mais je te félicite, au contraire. Tu as mené tout cela comme un ange.

- Cela m'a coûté cher, dit Perotto. J'ai été obligé d'apporter des gâteaux et du vin à la vieille et à sa cousine pour les mettre en confiance...

- Quelle nécessité de toujours me raconter des blagues? dit le cardinal. Dismoi donc simplement : “ Donnez-moi de l'argent. »

Il ouvrit son tiroir, en tira une pièce d'or, et la tendit à Perotto, qui la prit, et l'embrassa goulûment. Le cardinal eut une espèce de rire, en voyant ce geste, rire où il y avait du dégoût et en même temps de la sympathie. Habitué tout le jour à se contrefaire, il aimait tout ce qui était l'expression naïve d'un instinct.

Il regardait Perotto. Il n'y avait rien à reprendre au visage de Perotto pour en faire un visage digne d'entrer dans l'immortalité. On eût dit que les ciseaux du barbier n'avaient jamais touché sa nuque, tant les cheveux s'y arrêtaient naturellement, et exactement de la même façon que sur le buste bronze des Grecs. La peau était dorée, sans une faille, sans un " point noir » comme il y en a quelquefois dans la jeunesse ; les dents étaient virginales et on eût dit que la brosse n'y avait jamais touché ; la bouche était un peu lourde, d'un rouge un peu sombre, de framboise ${ }^{4}$. Pour la centième fois, il reconnaissait dans l'arc des sourcils, dans la lèvre supérieure, dépassant beaucoup l'inférieure, dans le dessus des oreilles, ses propres sourcils, sa propre bouche, ses propres oreilles, à lui, cardinal Capella. “Comment personne n'a-t-il jamais remarqué que mon page me ressemble? Tout au moins, je ne l'ai jamais entendu chuchoter. Et lui, comment ne s'en est-il pas aperçu? ”

- Remonte chez toi, lui dit-il, pendant que je vais m'habiller. Nous partirons dans une demi-heure.

Jamais le cardinal ne laissait Perotto seul dans son appartement du palais Ricardi, car Perotto y aurait volé quelque chose. Depuis sept ans que le cardinal Capella avait pris à son service ce petit apprenti forgeron, remarqué dans la rue, l'avait attaché à sa maison, puis à sa personne, Perotto avait volé pour des milliers de pièces d'or au cardinal. Il lui avait volé de l'or, des bijoux, des objets. Le cardinal, qui ne voulait pas paraître dupe, lui disait toujours quand il avait remarqué un vol.

4 Le portrait de Perotto fut ajouté dans la marge. 
Mais il ne le renvoyait pas, parce que Perotto lui était utile, ni même ne le punissait, parce qu'on ne peut pas punir quelqu'un qui vous tient. Le cardinal excusait tout avec cette phrase : “ Il faut bien qu'il ait intérêt à me servir. - D'ailleurs, si par hasard il est mon fils... »

Perotto parti, le cardinal entra dans sa garde-robe.

Après une demi-heure, il en sortit. Il était vêtu en homme de la toute petite bourgeoisie. Une courte barbe postiche encadrait son visage. Il avait sali ses mains avec de la suie.

Il alla à une armoire, y pris une custode, dont il sortit l'hostie, et l'enferma dans un petit sac d'étoffe, qu'il suspendit à son cou, sous son pourpoint. Le cardinal ne croyait ni à Dieu, ni au diable, ni à l'immortalité, ni seulement à l'âme. Mais quand il avait peur il croyait à n'importe quoi.

L'instant avant de quitter la pièce, il embrassa d'un coup d'œil son bureau, ses papiers. « Peut-être que je ne reverrai jamais tout cela », se disait-il.

Par un escalier dérobé, ils descendirent.

Quand ils furent dans la rue, selon leur coutume habituelle, le cardinal Capella marcha à une dizaine de pas derrière Perotto. Perotto, n’étant pas grimé, pouvait être reconnu, et les yeux alors se porteraient sur le compagnon du page du cardinal. Sans cesse le cardinal croisait de ravissants visages et à chaque fois, si violente était l'émotion qu'ils lui inspiraient qu'il crispait les mains, et, à voix haute, oubliant toute prudence, s'écriait : “Por dios ! Por dios ! » Comme saint François d'Assise, aux moments où il était ému, s'exprimait en français, le cardinal, sous le coup de l'émotion, reprenait d'instinct sa langue maternelle, le castillan.

Brusquement, devant une ravissante fille qui le croisait, et le dépassait, le cardinal pivota, fit demi-tour sur les talons, exactement comme eût pu faire une toupie, et resta figé, regardant la forme qui s'éloignait. «Me muero... disait-il, dans un souffle, me muero...5 ${ }^{5}$,

- Giorgio ! cria Perotto.

Le cardinal se retourna ; Perotto s'était arrêté et le regardait avec pitié, comme un enfant. Le cardinal fut péniblement affecté par cette familiarité. Dans leurs expéditions nocturnes, il lui avait été parfois utile, pour se camoufler mieux, de dire à Perotto de le tutoyer ; et maintenant, parfois, sans qu'il y eût nécessité, Perotto le faisait par badinage. Et le cardinal frémissait, songeant à ce qui se passerait $^{6}$ si Perotto, au palais, au cours d'une cérémonie, s'oubliait et lui disait $t u$.

5 Le manuscrit, ici comme plus loin, donne à lire Me muere, qui doit se corriger en $M e$ muero.

6 Le « passait » du manuscrit est sans doute une coquille. 
Maintenant, ils croisaient de moins en moins de monde ; aussi le cardinal s'était-il rapproché de Perotto, et ils marchaient côte à côte. À chaque fois qu'un homme émergeait de l'ombre, à l'improviste, tout auprès d'eux, le cardinal avait un rejet du corps en arrière, le réflexe de se mettre en garde.

Son regard tomba sur ses mains. Contre l'étoffe grossière qu'elles touchaient, la suie s'en était allée en partie. Il lui semblait que ses mains étaient révélatrices. Souvent il avait pensé que, dans le pire déguisement, le visage d'un homme ne trompe pas, et qu'un front habitué à la pensée, des yeux au mépris, une bouche au sceau des secrets profonds, jamais ne passeraient ${ }^{7}$ pour le visage d'un homme du peuple. Mais son visage était grimé et c'étaient ses mains qui, sales cependant, mais par leur seule forme effilée, eussent dénoncé un faux « petit bourgeois » à un œil inquisiteur ${ }^{8}$.

Parfois, à la dérobée, il regardait Perotto. Perotto marchait tête basse, le regard fixe, avec un visage dur, un si mauvais visage - cet enfant ! - que peu à peu le cardinal se sentait mal à l'aise. Mais ici, il faut quelques explications.

Quarante ans après l'époque où se passe ce récit, le chroniqueur Fiorentino écrivait, avec une force à la Tacite : « Le cardinal Capella haïssait l'univers entier. Perotto était son favori. Il le haïssait aussi. » Cela est tout à fait inexact. Le cardinal aimait Perotto. Mais comme c'était un homme emporté, il le haïssait par moments, sous le coup de la colère, quand Perotto lui tenait tête, quand il n'apportait pas toute la diligence qu'il eût pu aux singulières missions que lui confiait son maître, quand il apportait de mauvaises nouvelles, comme par exemple que telle personne faisait de la résistance, ou que de telle autre on avait perdu les traces, et qu'il ne pouvait la retrouver. Et aussi, par moments, le cardinal avait peur de Perotto. Singulier mouvement psychologique : de tout l'entourage du cardinal, celui en qui celui-ci avait le plus de confiance, c'était Perotto, qui le volait ouvertement. Il n'avait confiance ni en son honnêteté (bien entendu) ni en sa loyauté, mais il avait une sorte de confiance en son dévouement, et une confiance absolue en sa discrétion. Cet enfant de dix-sept ans était un tombeau de silence. Et cependant, nous l'avons dit, parfois il avait peur de lui. Il cachait tant qu'il pouvait cette peur, sentant bien que, si Perotto s'apercevait d'elle, elle ferait naître en lui la pensée d'actes que peut-être il n'imaginait pas. Et ainsi, cette nuit-là, voyant le visage sinistre de son page, une fois nouvelle lui apparaissait la pensée qu'il serait obligé un jour de le faire tuer et cette pensée lui fut pénible. Et il ajoutait : « Puisque moi, qui l'aime bien, j'en viens à cette pensée, lui, qui ne m'aime pas... ”Son visage devint plus sombre.

(Il s'arrête à la taverne) $)^{9}$

7 Le manuscrit donne à lire “ passerait ».

8 Tout ce paragraphe est sur un béquet.

9 Ajouté au crayon. 
Soudain une femme, avec un homme, entra et s'assit à une table.

Le cerne (naturel !) de ses yeux, - la rapidité de ses yeux se mouvant d'un point à l'autre, - la vivacité de sa façon de tourner les épaules, - comment elle se pliait en deux quand elle riait, - une espèce de vitalité convulsive, - comment elle s'essuyait la bouche avec un doigt, ou avec le dos de la main (Por dios!),-- son impossibilité de dire un mot sans avoir une espèce de frémissement, - comment elle ne pouvait rire sans porter la main à ses tempes, - comment elle repoussait ses cheveux en arrière, - la façon dont elle était toujours sur le bord de l'hystérie, sans y entrer jamais, - son renfoncement léger, entre les pommettes et les oreilles, comme si la mort avait légèrement pressé le visage, entre deux doigts, - ses mains, ses mains d'obscénité et de mensonge, - comment elle appuyait les doigts sur ses paupières pour montrer qu'elle était lasse (Por dios !), - comment elle se passait la langue sur ses lèvres, et dans les coins de sa bouche, quand elle avait mangé, - comment elle se mordait la lèvre inférieure, et ainsi, dans ce tic, sa bouche lourde, où le rouge (naturel !) débordait un peu tout autour, comme si on l'avait embrassée jusqu'à la faire déteindre - ses yeux! ses yeux ! ses yeux ! ses yeux ! - ce qu'il y avait d'effrayant en elle quand elle se mouchait, - comment elle se décoiffait, de la main, toujours un peu plus, à mesure que l'excitation du vin montait en elle.

Le cardinal était immobile, pétrifié, les bras écartés, tenant des deux mains, fortement, les deux bords de la table, qu'il pressait par instants convulsivement. Cette femme le poignardait, parce qu'il ne l'avait pas. Elle était la mort de ses jambes. Il ne pouvait plus bouger. Il lui semblait qu'il était Jésus Christ sur la croix. Ay, por dios! ou Yo me muero, répétait-il de temps à autre, à haute voix. S'il avait eu entre les dents un cigare, de passion, il l'eût sectionné, coupé en deux complètement ; mais il n'y avait pas encore de cigares, ils ne devaient être inventés que deux siècles plus tard. Il n'y avait que ses mains qu'il n'aimait pas, - des mains de femme, et le cardinal Capella n'aimait que les mains de garçons.

Par instants le cardinal posait ses yeux sur le compagnon de la femme. Sans être à proprement parler immonde, il était ordinaire, et - du moins le cardinal le jugeait ainsi, - beaucoup moins bien que lui. La femme était amoureuse de lui, cela se voyait bien. Et malgré le regard fixe du cardinal posé sur elle, et ses Por dios à voix haute, pas une fois elle n'avait seulement rencontré son regard. « Pourquoi aime-t-elle cet homme? se disait le prince de l'Église. Un hasard a fait cela. Un hasard eût fait que c'eût été moi. Je suis venu trop tard. » L'idée que cette femme pût désirer un autre homme que lui paraissait au cardinal Capella une chose si incroyable, si inadmissible, si contre-nature qu'elle lui apparaissait comme un jour où la lumière du soleil luirait pendant vingt-quatre heures.

En vain Perotto lui disait-il qu'ils allaient manquer leur rendez-vous. " Mais non, mais non ", disait le cardinal, qui savait bien que tout le monde était toujours en retard. Il était stupéfié. Si son regard s'égarait sur les autres femmes, il fermait les yeux quand il les rencontrait tellement, elles lui paraissaient laides. À ses 
Por dios! avait succédé une sorte d'onomatopée, $m a-m a-m a-m a-m a$ (peutêtre le début de l'interjection Madonna!) sur un ton decrescendo, qui exprimait, dans l'esprit du cardinal, tout le désespoir où il était de ne pas posséder cette femme. Maintenant il ne la regardait même plus. Il regardait fixement autre part, incapable de s'en aller, et goûtant singulièrement que ces précieux instants, qui peut-être lui faisaient manquer son rendez-vous, fussent perdus en vain, puisqu'il ne contemplait même pas, et ne répondait que par un non de la tête quand Perotto lui faisait : “ Alors? ” Brusquement il s'arracha, cracha dans son verre, de dégoût, paya le gargotier, et sortit, sans avoir plus une fois regardé la belle.

Le cardinal et Perotto sortirent de la maison, rasant les murs. La nuit était encore toute noire. Comme pour venir Perotto marcha en avant. Sans raison, à l'instant où il allait tourner dans une ruelle, le cardinal se retourna, et il vit trois hommes, quatre peut-être s'arrêter devant la porte de la maison qu'il venait de quitter. Des sbires? Des complices de la personne qu'il venait de voir, qu'elle venait de prévenir, et qui arrivaient quelques secondes trop tard? "Cours!" jeta-t-il à Perotto. “ Pourquoi ? » dit Perotto. Il ne répondit pas et se mit à courir. Perotto courait en avant. Quand l'essoufflement arrêta le cardinal, et le supplice de ses jambes affaiblies par la volupté récente, et chancelantes sur le dur cailloutis raboteux, Perotto lui demanda encore : “Qu'y a-t-il ? » Il dit les hommes. Perotto haussa les épaules. “Vous avez peur de tout. » “ Et ma peur est fondée, dit le cardinal, avec impatience. Je sais ce que tu ne sais pas. » À la vérité, il ne savait rien que ne sût Perotto, mais il se cherchait une excuse.

- Vous y reconnaissez-vous, maintenant? Pouvez-vous rentrer seul ? demanda Perotto.

- Non, je me perdrai. Ne peux-tu m'accompagner jusqu'aux abords du palais?

- C'est que moi aussi il faut que je sois rentré à huit heures. Et, auparavant, j'ai à faire.

- Rentre plus tard.

Perotto se mit à rire.

- Vous oubliez que c'est moi qui vous sers la messe à huit heures !

- Comment, c'est toi !

- Naturellement. C'est mon jour. Et, avant de rentrer, j'ai quelque chose à faire pour moi.

De nouveau une onde de défiance traversa le cardinal. Qu'avait-il à faire ? Quoi de louche? Ah, jamais, jamais, jamais un instant paisible d'absolue sécurité.

- Accompagne-moi encore un peu. Jamais je ne me reconnaîtrai pour revenir.

Après quelques instants, Perotto s'arrêta.

- Il faut que je vous quitte. Prenez par ici, par là (il lui expliqua le chemin). Adieu. 
Le cardinal marcha seul, dans la direction indiquée. Il lui semblait que, physiquement, il tenait la tête plus haute qu'en venant. La chose était passée, mise derrière soi. Il se sentait plus pur, allégé. Brusquement, dans son chemin, il ne se reconnut plus.

La terreur le saisit. Il se vit perdu, n'arrivant au palais que le jour levé, dans la ruelle populeuse où des gens le reconnaitraient, s'inquiéteraient de cet homme inconnu qui avait une clef de la porte basse. Mais l'avait-il, seulement, cette clef? Ne l'avait-il pas laissée là-bas? Fébrilement il la tâta dans sa poche. Alors un nouveau fantôme se leva en lui. L'agonie, sur son seuil, si la clef ne prenait pas, si cela faisait comme cela avait fait à la maison.

Le cardinal tournait sur lui-même, à un carrefour, comme une bête dans une cage. Les ruelles étaient ouvertes de tous côtés, mais c'était comme si elles avaient été fermées. Il vit venir un homme, et pensa lui demander son chemin. Mais sitôt que l'homme fut plus rapproché, la peur fut plus forte, il se rencogna dans un renfoncement.

Il lui sembla que le ciel, du côté du levant, était plus clair. Elle s'ouvrait la lutte pour gagner de vitesse le jour ! Le cardinal marchait égaré, sans savoir s'il ne tournait pas le dos à son palais. Un autre homme apparut. Il eut le courage de lui demander, et repartit dans une direction nouvelle.

Maintenant, des passants naissaient, ouvriers remplaçant les équipes qui avaient travaillé la nuit sur le Tibre. Rome était une ville d’hommes.

\section{Les notes}

Les deux pages de notes écrites à même la lettre des Éditions du Capitole du 27 février 1930 permettent, si décousues qu'elles soient, de mieux évaluer ce qui nous est parvenu du Cardinal Capella. Les voici donc dans leur intégralité ${ }^{10}$.

La terreur de la maison du retour, de l'insulte ou de graffitis obscènes dessinés sur sa porte, du billet de menaces glissé jusque sous la porte de sa chambre, de la mauvaise nouvelle, qu'on lui annoncerait, de quelque chose qui s'était passé en son absence.

Matin, le retour de $<$ ces q $>$ hommes, $<$ prient $>$, yeux khol

cf. Syracusaine

le matin se met du carmin sur les joues, de la poudre sur les paupières et les cernes

10 Quelques mots d'une lecture incertaine seront entre chevrons. On rappelle que c'est en travers de la première page de notes que Montherlant a écrit en grand “ CAPELLA ». Il a aussi écrit dans le coin supérieur gauche de cette page le mot “ divers » souligné trois fois. 
il essaya de ragaillardir son visage $\mathrm{p}^{\mathrm{r}}$ lui donner un air insouciant $\mathrm{cf}$. XII. 155 vermine [au crayon]

ill. esprit perdu [au crayon]

toutes les fois qu'il lui indiquait des ruses, lui faisait la leçon, il se disait : « Je prépare des armes contre moi. »

début : conversation importante

temps infini pour faire entrer la clef dans le pêne, se disant que tout va manquer à cause de cela

attendant, souhaite qu'elle ne vienne pas ${ }^{11}$

sa faim de travail

tête haute

boire vin

la colline d'or

souhaitant mort, ou la meute des gens qu'il a mis contre lui et des périls nés de ses infractions s'arrêtera contre les portes sombres du néant

la peur d'arriver au jour

il était purifié, - mais un acte de plus restait, ineffaçable

itl. hostie après double sacrilège (il a bu) qui mit en lui une brûlure qui fut autre que celle de son âme

Le cardinal voyait parfois comme une colline d'or le monceau d'or qu'il avait dépensé pour ses plaisirs (comme une colline < précieuse > sous le soleil) Il y avait des vieillards etc... Alors le cardinal était triste, songeant à quelle épreuve c'est, de devoir reconnaître que l'argent ne peut pas tout.

Si on lui disait que telle personne n'était pas à Rome, il en déduisait qu'elle y était sûrement. Si tel lui écrivait : “Je vous écris au crayon, pcq. je suis au lit, malade », il pensait que ce n'était pas vrai. Perotto lui dit : «La patronne d’une de vos maisons a dit que vous aviez l'air d'un drôle de bonhomme. » Le cardinal est sombre. En vain se dit-il : “C'est ma barbe... », il se demande si ce n'est pas sa voix etc... enfin son identité même, et il est froissé. Alors il aspire vers une vie sans dessous, où on soit tjrs naturellement respectable, qu'on puisse regarder les gens en face. Mais il était trop engagé ; il n'aurait plus jamais cette vie-là, maintenant.

Le lecteur aura compris pourquoi il valait mieux lire les notes après le texte. Si la “ conversation importante » du début, les efforts que déploie le cardinal pour se donner l'air insouciant, “ la peur d'arriver au jour » se retrouvent plus ou moins dans les pages qu'on vient de lire, le maquillage du matin en revanche, la tristesse de constater que l'argent n'achète pas tout, le pli de toujours soupçonner qu'on vous ment, l'aspiration à une vie « sans dessous », etc. sont destinés à d'autres pans

1 Entre cette note et la suivante, Montherlant a écrit un « T.S.V.P. » par lequel il signale, semble-t-il, à sa propre attention les deux fragments plus élaborés qu'il a rédigés au recto de la lettre. 
du récit ${ }^{12}$. Même chose avec “ temps infini pour faire entrer la clef dans le pêne, se disant que tout va manquer à cause de cela », par quoi s'explique l'angoisse du retour : “ L'agonie, sur son seuil, si la clef ne prenait pas, si cela faisait comme cela avait fait à la maison ». On ne sait d'ailleurs pas pourquoi le cardinal a fait halte à la taverne (parce qu'il avait la gorge sèche ou pour s'encanailler ?) et si on a bien compris qu'il allait à un rendez-vous amoureux (car le mal qu'il aura, au retour, à suivre Perotto n'est pas que le fait de son âge, il résulte aussi du plaisir qu'il vient de prendre), on ignore comment les choses se sont passées dans la maison où il avait son rendez-vous et quelle sera l'issue de cette équipée nocturne. Ce qui, admis qu'il s'agit d'un texte lacunaire, appelle aussi quelques remarques touchant la date de Capella.

S'il n'y avait que la lettre des Éditions du Capitole qu'on a citée plus haut, on dirait sans hésiter que Capella fut écrit en 1930. Sauf que les versos du texte sont tous antérieurs au 27 février 1930. Les neuf premières pages, rédigées au dos de placards de La Relève du matin ou de Pages de tendresse ${ }^{13}$, ne nous instruisent guère, mais les dix autres pages, qui furent toutes rédigées au dos de lettres se situant entre le 4 septembre 1929 et le 10 février 1930 ${ }^{14}$, invitent à la prudence.

12 Si la référence « cf. XII. 155 » reste un mystère, la notation “ cf. Syracusaine » est moins étrange qu'il ne paraît, dont le point de départ est un " modèle » que Montherlant connut en 1913 quand, à l'insu de ses parents, il loua une chambre d'hôtel au 43 de la rue Vavin pour y recevoir lesdits modèles. Non seulement la Syracusaine a laissé de nombreuses traces dans l'œuvre, dans Le Démon du bien notamment (voir le vol. Romans 1 de la Bibl. de la Pléiade, p. 1304, R1 désormais), mais il ressort clairement de certains feuillets du fonds Montherlant du Musée Richard Anacréon de Granville que Montherlant avait envisagé, peu après 1925, d'écrire un roman ou un récit intitulé $L a$ Syracusaine. Quand on se rappelle l'épisode de la taverne dans Le Cardinal Capella, on est moins étonné de cette allusion à la Syracusaine.

13 On lit « La Relève Placard 16 » au dos de la p. 3 du texte et « Pages Placard 12 » au dos de la p. 9. Les pages 3, 4 et 5 pourraient correspondre à l'édition Spes de La Relève, dont l'achevé d'imprimer est du 31 décembre 1928. Pour les pages 1, 2, 6, 7, 8 et 9, en revanche, il n'y a pas à hésiter ; elles correspondent aux pages 76-77, 78-79, 102-103, 96 à 98, 98 à 101 et 93 à 96 de Pages de tendresse, une espèce d'anthologie que Montherlant fit paraître chez Grasset en 1928 pour corriger l'image qu'avait de lui le public.

14 En voici le détail. Trois courriers envoyés par L’Argus de la Presse les 13 et 17 décembre 1929 et le 3 janvier 1930 ont servi pour la page 11 de la première partie ainsi que pour les pages 1 et 2 de la troisième partie. La page 1 de la seconde partie est au dos d'une lettre de Grasset du 14 janvier 1930 avisant Montherlant que l'éditeur a déclaré au contrôleur des contributions lui avoir versé la somme de 58.934,35 frs pour l'année 1929. Une lettre du 4 septembre 1929, signée d'Harold J. Salemson, directeur de Tambour, a servi pour la page 2 de la seconde partie. La page 3 de cette même seconde partie est au dos d'une lettre du 4 septembre 1929 par laquelle l'éditeur Insel-Verlag 
On a constaté que la substance des notes ne se retrouve guère dans le texte et par ailleurs on sait que Montherlant avait pour habitude de barrer les notes dont il avait fait usage, or il n'en a barré cette fois-ci aucune. Il sera donc plus sage de dire que Le Cardinal Capella se situe à cheval entre la fin 1929 et le début 1930, les notes préparant une nouvelle étape dans la composition.

\section{La place du Cardinal Capella dans l'œuvre de Montherlant}

Pour bien situer Le Cardinal Capella dans l'œuvre de Montherlant, il faut remonter à la mi-février 1929, quand l'écrivain quitte Alger, où il réside depuis octobre 1928, pour regagner la France via le Maroc et l'Espagne. C'est alors en effet que, s'étant arrêté trois jours à l'abbaye de Montserrat, il y « rencontre un moine qui fait tous les gestes de la foi, mais qui ne croit pas ». Or si de l'avis général, « ce moine est à l'origine du personnage de l'abbé de Pradts dans La Ville dont le prince est un enfant et dans Les Garçons ${ }^{15}$ ", il pourrait avoir aussi déteint sur un cardinal

de Leipzig annonce à l'écrivain l'envoi de deux exemplaires de l'édition allemande des Bestiaires. La page 4 enfin est au dos d'une lettre du 12 janvier 1930 où le directeur des Cahiers idéalistes, Édouard Dujardin, annonce à Montherlant son intention de traiter, dans une série de conférences qu'il donnera dans plusieurs villes d'Allemagne et sous la forme d'articles qui paraîtront dans des journaux de Berlin et de Munich, d'un sujet qui divise les écrivains allemands et français : “ Tendances de la jeune littérature française relativement aux questions qui se posent entre la France et l'Allemagne ». Dujardin demande à l'écrivain de « formuler, aussi brièvement que possible, [ses] vues sur la question » et de lui indiquer, parmi ses écrits, les pages qui lui semblent le mieux illustrer son point de vue. Un embryon de réponse fut d'ailleurs griffonné sur la lettre même de l'expéditeur : “ Devant l'opinion française on met, avec assez d'insistance, l'accent sur le mot "rapprochement", pour qu'il n'y ait pas un devoir à le mettre aussi, un peu plus, sur le mot "prudence" ». On sait déjà que deux courriers de L'Argus ont servi pour les pages 1 et 2 de la troisième partie ; reste, pour les pages 3 et 4 , un long courrier de Paul Vanderborght, directeur de la revue belge La Lanterne sourde, daté du 9 février 1930. Et si on a réservé pour la fin la page 10 de la première partie, rédigée au dos d'une facture (4.300 frs) envoyée par Carette, tailleur de luxe du boulevard Haussmann, c'est que ladite facture porte la date du 10 février 1930, qui est donc la plus récente pour ce qui est du texte.

15 On a repris la formulation de la “ Chronologie » mise en tête du vol. Romans II de la Bibl. de la Pléiade (R2 désormais), p. L, mais il faudrait la nuancer. Que ce soit dans la version définitive de Trois Jours au Montserrat (R1, p. 389-390) ou dans sa forme primitive (Pour une Vierge noire, dans le recueil du même nom paru en 1930 aux Éditions du Cadran, p. 39, voire dans l'extrait qu'il en publia sous le titre " Trois Jours au Montserrat " dans Les Nouvelles littéraires du 3 mai 1930, p. 9), Montherlant distingue le Père Abbé de l'abbaye, de qui on lui dit : « [...] croit-il : $1^{\circ}$ à l'âme ; $2^{\circ}$ à Dieu ; $3^{\circ}$ à la divinité de 
qui « ne croyait ni à Dieu, ni au diable, ni à l'immortalité, ni seulement à l'âme », même si « quand il avait peur il croyait à n'importe quoi ».

Quoi qu'il en soit, Montherlant consacre le reste de l'année à son œuvre. La petite Infante de Castille est déjà derrière lui, dont l'achevé d'imprimer est du 31 janvier, mais il publie trois recueils de textes, Sous les drapeaux morts ${ }^{16}$, Le Génie et les fumisteries du Divin et Hispano-moresque, ainsi que L'Exil, une pièce écrite en 1914, tout en travaillant sur Moustique ou l'Hôpital, un roman qui ne parut, incomplet, qu'en 1986, sur Don Fadrique, une pièce commencée en 1928 qu'il abandonne pour de bon dans le courant de 1929, sur La Ville dont le prince est un enfant, qu'il laisse en suspens, et sur Les Garçons, la version romanesque de La Ville, dont il rédige cinquante pages, sinon plus.

L'année 1930, moins riche en publications (ne paraîtront que deux recueils d'essais, Pour une Vierge noire et Au petit Mutile), ne fut pas moins féconde : Montherlant, après avoir œuvré au premier tome des Jeunes Filles ${ }^{17}$, repart le 19 septembre pour Alger, où il commence d'écrire La Rose de sable. Notre Capella s'inscrit donc dans une période de travail intense mais peu « rentable » : beaucoup d'œuvres mises en chantier et laissées en plan.

Que dire de ce cardinal hors du commun?

S'il faut trouver un Espagnol de sa famille dans l'œuvre de Montherlant, ce n'est sûrement pas au maître de Santiago des années 1945-46 qu'on va de prime abord penser, ni au cardinal Cisneros des années 1957-58, non plus qu'à Celestino Marcilla, l'anarchiste républicain du Chaos et la nuit des années 1961-62. On pensera plus volontiers à Don Juan, né comme Cisneros en $1957^{18}$, à cause de ses fredaines, de ses prodigalités, de son incrédulité, ou alors, pourvu qu'on quitte l'Espagne, à Sigismond Malatesta, qui avait vu le jour en 1943, ou du moins à l'Italie qui fut la sienne.

Car on est bien, avec ce Giorgio Capella aux nom et prénom italiens, dans l'Italie de la Renaissance, on est même à Rome, malgré ce palais Ricardi ${ }^{19}$ transplanté de Florence où vit le cardinal, et l'air qu'on y respire est un peu celui qu'on y respirait - revoilà donc l'Espagne - sous les Borgia. L'auteur de Malatesta, ne l'oublions pas, campera parmi les cardinaux entourant Paul II l'Espagnol Rodrigue Borgia, alors

Jésus-Christ? », du « Père Abbé de tel monastère (non espagnol) » dont on lui rapporte qu'il « est présumé n'avoir pas la foi ».

16 Rappelons-nous la lettre des Éditions du Capitole.

17 Qui ne sera cependant publié, après remaniement, qu'en 1936. Qu'on y ajoute foi ou non, c'est un fait que l'édition originale des Jeunes Filles est datée « Alger, 1930 » et que l'Avertissement commence par les mots “Ce roman a été écrit en 1930 ».

18 Voir la postface du Cardinal d'Espagne aux p. 1171-1173 dans le vol. Théâtre de la Bibl. de la Pléiade.

19 La graphie Ricardi avec un « $\mathrm{c}$ » fut autrefois en usage. 
âgé de trente-sept ans, qui coiffera la tiare en 1492 sous le nom d'Alexandre VI. Lequel Alexandre va placer auprès de sa fille Lucrèce quand elle sera plus ou moins recluse au couvent de Saint Sixte dans l'attente d'un second mari, un camérier tout de même espagnol, un certain Pedro Calderon surnommé... Perotto. On a beaucoup rêvé autour de ce Perotto ${ }^{20}$, dont le cadavre fut retrouvé sur les rives du Tibre le 14 février $1498^{21}$, et Dieu sait quel sort Montherlant réservait au page de Capella, mais il est évident que le bâtard du cardinal tire son nom de la chronique ${ }^{22}$.

Autre affinité entre Alexandre VI et Capella : l'hostie. Alexandre, depuis qu'un astrologue lui avait prédit qu'il échapperait à la mort aussi longtemps qu'il aurait avec lui une hostie consacrée, ne se séparait plus, dit-on, de son " talisman ». Gobineau s'en était souvenu dans ses Scènes historiques, qui venaient d'être rééditées en $1929^{23}$, et il n'est pas impossible que Montherlant en ait parlé avec son ami Faure-Biguet, celui-là même qui avait publié en 1925 un Montherlant homme de la Renaissance, qui publiera en 1941 Les Enfances de Montherlant et qui achevait un Gobineau dont Montherlant rendra compte, avec chaleur, dans Les Nouvelles littéraires du 6 septembre 193024. L'hostie de Capella en tout cas, d'où qu'elle vienne, est un souvenir de celle du pape Borgia ${ }^{25}$.

20 Peroso chez Michelet.

21 Il aurait été le père de l'enfant que Lucrèce mit au monde un mois plus tard, l'Infans Romanus que par deux bulles successives Alexandre VI reconnut d'abord comme le sien, puis comme celui de son fils César. Ferdinand Gregorovius, l'auteur d'un Lucrèce Borgia resté fameux qui parut en 1876, chez Sandoz et Fischbacher, dans la traduction de Paul Regnaud, ne va pas jusque là, mais il impute l'assassinat de Perotto à César Borgia et cite le Journal de l'ambassadeur de Venise, Polo... Capello, où on lit que César poignarda le camérier « sous le manteau du pape » (p. 239 et 326 respectivement).

22 Les lecteurs de Thrasylle, roman de jeunesse de Montherlant (il se situe entre fin 1912 et 1915), se rappellent que Thrasylle fut un temps apprenti forgeron, comme ici Perotto.

23 “Son hostie! son hostie ! Depuis qu'on lui a assuré que, tant qu'il l'aurait sur lui, il ne pouvait lui arriver malheur, s'il la perd de vue, il devient fou ", s'écrie dans les instants qui précèdent la mort d'Alexandre le valet Caraffa que son maître a chargé d'aller chercher de toute urgence la boîte où il serre cette hostie et qu'il a par mégarde oubliée (voir La Renaissance Scènes historiques, Nouvelle édition, Plon, 1929, p. 273).

24 L'article, qu'on lit en page 1, s'intitule « Sur une vie de Gobineau. De l'esprit lyrique à l'esprit critique $"$.

25 En 1929 avait aussi paru chez Payot l'ouvrage de Giuseppe Portigliotti, Les Borgia, dans la traduction française de Fernand Hayward, et Perotto y est plusieurs fois cité, ainsi d'ailleurs que le saint François Borgia dont parle Montherlant dans La grande Tentation (p. 623 dans le vol. Essais de la Bibl. de la Pléiade, E désormais). Il est aussi question dans La grande Tentation du film Lucrezia Borgia (et non César Borgia) 
Cardinal dans la meilleure tradition des prélats de la Renaissance italienne, Capella est aussi parent de ces deux “ chasseurs » à qui Montherlant donnera bientôt le jour, à savoir le Costa des Jeunes Filles ${ }^{26}$ et le Guiscart de La Rose de sable . S'il n'est question pour Costa que d'un unique bâtard ${ }^{27}$, Guiscart ne compte pas moins de quatre « foyers » autour de la Méditerranée, et « il y avait eu une période de sa vie où il nageait parmi ses bâtards comme Tibère parmi ses pisciculi ${ }^{\mathbf{2 8}}$ ». En quoi du reste il annonce Don Juan, qui parlera de son “ peuple » de bâtards et de bâtardes ${ }^{29}$.

Autre point commun entre Capella et nos trois libertins, la prodigalité quand il s'agit d'assouvir ses plaisirs et de régaler ses proies, et c'est le traqueur des Jeunes Filles, cette fois-ci, qui s'apparente le plus au prélat. Déclarant à sa maîtresse : “Quand je serai vieux et misérable [...],je rêve que tout l'argent que j’aurai dépensé pour les êtres que j'aimais se reconstituera quelque part, sous une forme tangible, et que je m'en irai content de ce que j'ai fait, les yeux fixés sur cette montagne d'or, - d'un or que, sans vouloir vous choquer, j'appellerai l'or du rein ${ }^{30}$ », Costa n'est pas loin de Capella qui « voyait parfois comme une colline d'or le monceau d'or qu’il avait dépensé pour ses plaisirs ».

Autre trait encore par quoi Giorgio Capella se rapproche de ses compères, il préfère ses passions à sa tranquillité. Quand pour les assouvir il se lance dans de périlleuses expéditions nocturnes, il annonce Don Juan revenant à Séville où ses “ caracoles » lui “ ont créé mille ennemis ${ }^{31}$ ».

Il s'éloigne, en revanche, de Don Juan quand il se vêt “ en homme de la toute petite bourgeoisie ", s'arrange une barbe postiche, se salit les mains de suie, son but n'étant pas de camoufler son âge comme prétend le faire Don Juan lorsqu'il

tourné en 1922 par l'Allemand Richard Oswald, avec Conrad Veidt dans le rôle de César, autre indice de l'intérêt que Montherlant a porté aux Borgia (E, p. 622). On relève enfin dans la version définitive de Trois Jours au Montserrat, sinon dans les versions de 1930, un ironique "Cette famille Borgia savait bien faire les choses », pour commenter l'institution de l'Angélus, ou du moins du triple Angélus, par Calixte III, oncle maternel d'Alexandre VI (E, p. 389), et ce mot du héros des Jeunes Filles sur « le Borgia Alexandre VI, un peu personnel pour les mœurs, mais qui sur la doctrine n'avait jamais fléchi d'un pouce » (R1, p. 1270).

26 Si on admet que Les Jeunes Filles furent commencées dès 1930, le héros devait alors porter le nom qu'il portera lors de la parution des Jeunes Filles.

27 Prénommé Philippe et surnommé Brunet, il apparaît à la p. 134 de l'éd. originale des Jeunes Filles (R1, p. 989).

28 R2, p. 148.

29 Voir La Mort quifait le trottoir (Don Juan), III, I.

30 Pitié pour les femmes, p. 172 de l'éd. originale (R1, p. 1165-1166).

31 En I, I. 
rabat un masque d'étoffe sur son visage ${ }^{32}$, mais de s'assurer l'anonymat, et c'est alors le libertin de La Rose de sable qui se profile derrière notre Capella - sinon, à y bien réfléchir, cet abbé de Pradts de La Ville et des Garçons qu'on a évoqué plus haut.

Collaborateur zélé de la revue Dieu vivant, de Pradts est le parfait hypocrite à la conscience tranquille : “ Il n'était pas sincère. Mais pourquoi aurait-il fallu être sincère ? [...] Qui ne portait pas de masque, hormis les simples d'esprit ? Il était comme les autres. Et sans faire de mal à quiconque ${ }^{33}$ ». Entre Capella et de Pradts, il y a plus que des nuances, mais le " péché », tout bien considéré, n'est pas moins grave chez l'abbé que chez le cardinal, la différence tenant d'abord à ceci que l'un porte son masque - rien moins qu'une soutane - avec une parfaite assurance, quand l'autre craint à chaque instant de se trahir ${ }^{34}$.

On ne peut d'ailleurs pas s'empêcher, quand on est dans ces registres, de penser à Montherlant lui-même, qui, par jeu, s'affuble, au carnaval de Binche de 1938, d'une " tête de taureau en carton ${ }^{35}$ ", qui, plus gravement, se fait photographier avec, devant son visage, « le masque de Conflans », cette visière de casque d'officier romain qu'il avait prévu d'emporter dans la tombe (ce qui ne fut pas $)^{36}$, mais qui, par ailleurs, voyage volontiers sous son patronyme, M. Millon, et pousse un cri d'allégresse qui n'est pas qu'une boutade lorsqu'il découvre, par un beau jour de 1937, qu'un journal a mis son nom sous une photo qui n'est pas la sienne : “ Inondation de possibles! Combien de tromperies en perspective ! Sancta securitas $!^{37}{ }^{3}$

32 Et dont le seul effet est d'effrayer la jeune fille qu'il convoite (en I, II).

${ }^{33}$ Les Garçons, R2, p. 588. Faut-il rappeler que le mot hypocrite, en grec, désigne l'acteur et que l'acteur porte un masque?

${ }^{34}$ Il vaut la peine de préciser que, près de mourir, l'abbé de Pradts des Garçons devient “tout de bon l'homme de foi qu'il était censé être»(R2, p. 836). Montherlant, énumérant les trois raisons qui le poussent à “ se convertir », est d'ailleurs revenu sur “ la double et la triple duplicité de l'abbé de Pradts, qui feignait d'être gouvernemental et était conservateur, qui feignait d'être droit avec les jeunes et était avec eux un peu de biais, qui était ministre de Dieu et ne croyait pas en Dieu ", non sans se demander si “ par l'excitation d'esprit, l'intelligence, la vigilance, l'amusement qu'elle donne », la duplicité ne serait pas " en fin de compte plus ou moins garantie de jeunesse prolongée » (R2, p. 835).

35 La Marée du soir, Paris, Gallimard, 1972, p. 11.

36 Voir, sur le site de l'Ina.fr, l'interview que Montherlant accorda le 9 février 1954 à Pierre Desgraupes pour son émission Lectures pour tous.

37 E, p. 1218. Le conseil d'autarcie que donnait Montherlant en 1934 : “Vis seul, tu donneras moins de prise qu'entouré d'une garde du corps ; moins de prise qu'entouré d'amis » (E, p. 1129), est aussi un conseil de prudence. 
Guiscart, qui se fait appeler Destouches ${ }^{38}$ et chez qui la peur d'être reconnu tourne à l'obsession, va plus loin. Quand on fait sa connaissance, il a le bras gauche en écharpe pour une blessure feinte et se dissimule derrière un journal afin d'échapper aux regards d'un passant, non sans prétendre, dans l'instant même où il admet qu'il a pâli, qu'il n'a pas peur. Mieux, on le voit peu après qui " détache d'un complet neuf ces damnées petites étiquettes de toile où les tailleurs inscrivent à l'encre votre nom, et qui sont si propres à vous trahir dans les circonstances délicates ${ }^{39}$.

Toutes ces précautions, on l'a bien vu avec Capella, ne protègent pas ipso facto de la peur. Or avec la peur, on change de registre ; on passe du ridicule au tragique. Si les ruses de Guiscart font sourire, parce que l'auteur de La Rose de sable les a traitées sur le mode de la caricature, Capella étreint par la peur se hisse au niveau de ce Celestino Marcilla, pathétique héros du Chaos et la nuit, qu'on ne pensait pas, de prime abord, rapprocher du veule Capella.

Anarchiste vivant en exil à Paris depuis dix-huit ans, Celestino vient d'hériter de sa sœur, restée à Madrid. La question est de savoir s'il n'est pas dangereux pour lui de rentrer dans une Espagne toujours franquiste, et dès cet instant la peur, une certaine peur, s'empare de Celestino. Cet homme de soixante-sept ans qui s'amuse à “ toréer » les automobiles en plein Paris, était celui, nous dit le narrateur, “ qui dans le même temps avait si peur, et surtout allait avoir si peur, d'aller à Madrid ${ }^{40}$ ». La gare d'Austerlitz sera pour lui “ la gare de la mort » et sa patrie d'origine, " le pays de la peur ", de " la pâle peur, aussi subite et brutale qu'une crise cardiaque ou une attaque d'épilepsie ${ }^{41}$ ».

38 Inspiré du nom de la grand-mère paternelle de Montherlant : Marie Bessirard de La Touche (1843-1870).

39 La Rose de sable, R2, p. 11, 13, 20 et 26 respectivement. Et puisqu'on est dans les déguisements, on ne peut passer sous silence ce Don Fadrique que Montherlant avait créé en 1928 pour l'abandonner en 1929, assurément pas le Fadrique que tout le monde connaît par le court dialogue repris dans Service inutile en appendice à La grande Tentation, mais celui qu'on découvre, après Capella, dans le même dossier NAF 28165, boîte 16 (Varia): Textes des années 1929-1930, et pour lequel, d'ores et déjà, on prend date avec le lecteur. Fadrique, dans ce manuscrit, se déguise à deux reprises : une première fois en bourgeois pour se faire accepter au monastère où il veut entrer incognito (et quand son intendant lui a demandé pourquoi il agissait de la sorte, il a répondu : “ J'aime me sentir inconnu et libre »), une seconde fois en paysan, par simple nécessité, quand il a jeté son froc aux orties.

40 Le Chaos et la nuit, R2, p. 948.

41 Ibid., p. 972 et 973 respectivement. Louis Baladier a bien mis en évidence cette peur de Celestino dans "Le Chaos et la nuit : une poétique originale 》(dans Actualité(s) de Montherlant, Babel, Université du Sud Toulon-Var, 27-2013, p. 211-223). 
Tout est réglé en un tournemain ou presque, il aura bientôt quitté Madrid, mais rien n'y fait : « La peur d'être arrêté, la peur moins de la mort que de l'avant-mort et des conditions matérielles qui l'accompagneraient, [...], la conscience qu'il était déjà à demi mort puisque la réalité ne parvenait plus à l'accrocher [...] le menaient à un point d'angoisse où ses jambes flageolaient presque sous lui ${ }^{42}$ ", comme sous Capella quand il est « sur le dur cailloutis raboteux » du retour.

La peur de Celestino était justifiée, puisque la police qui découvre son corps sans vie dans sa chambre d'hôtel venait en fait pour l'arrêter, mais qu'il s'agisse de Celestino, dont nul ne songerait à se moquer, ou de tout autre, brave homme ou franc scélérat, on en retient qu'aux yeux de Montherlant, la peur n'a pas besoin d'excuses pour être excusée. Quand à l'époque du Treizième César il évoquera “l'épouvante de Néron et sa fuite hagarde », il n'aura de mots que charitables : "L'homme que nous avons vu une fois épouvanté doit nous devenir sacré, que son épouvante ait été justifiée ou non ; sa crise d'épouvante a pour moi le même caractère semi-religieux qu'avait pour les Latins le morbus sacer, l'épilepsie ${ }^{43}$. »

L'image du prince épouvanté vient à propos nous rappeler que la “ virée » nocturne de Capella tient plus ou moins des incartades que Suétone - dont il est inutile de rappeler qu'il fut très tôt parmi les livres de chevet de Montherlant ${ }^{44}-\mathrm{a}$ mises sur le compte de Néron ou de Caligula : Néron “ attrapant, la nuit venue, un bonnet ou une casquette pour aller dans les cabarets et traîner dans tous les quartiers de la ville en faisant mille folies ${ }^{45}$ », Caligula “ courant la nuit à la débauche et à l'adultère coiffé d'une perruque et vêtu d'un long manteau ${ }^{46}$ ». On sait bien qu'il n'est question dans Le Cardinal Capella que de Tacite, et par le biais d'un chroniqueur imaginaire ${ }^{47}$, mais comment se soustraire à Suétone quand on a lu ce qu'on vient de lire du Néron fugitif dans Le Treizième César et qu'on revoit Capella, hagard lui aussi dans la Rome du petit matin? Le proprium quid du cardinal Capella, et qui donne le ton au récit, ce n'est pas tant qu'il ait des maîtresses ou s'encanaille dans les tavernes, c'est la peur : peur d'être démasqué par le premier

42 Ibid., p. 1010-1011.

43 Le Treizième César, Paris, Gallimard, 1970, p. 158-159, et aussi, presque dans les mêmes termes, dans une note de Tous Feux éteints, Paris, Gallimard, 1975, p. 165. Par ailleurs, on vient de voir que dans Le Chaos et la nuit, " la pâle peur » est rapprochée, par sa soudaineté et sa brutalité, d'une attaque d'épilepsie.

44 Le pisciculi de La Rose de sable nous l'a rappelé, qui vient de la Vie de Tibère (XLIV, 1).

45 Un jour que Néron avait serré de près la femme d'un sénateur, celui-ci, ajoute Suétone, réagit si vigoureusement que le prince faillit y laisser la vie, de sorte que depuis ce moment-là il se faisait suivre discrètement par des gardes du corps (Nero, XXVI, 1-4).

46 Cal., XI, 1.

47 Il n'y eut pas, qu'on sache, de chroniqueur appelé Fiorentino. On connaît le poète Giovanni Fiorentino, mais qui vécut au $\mathrm{XIV}^{\mathrm{e}} \mathrm{s}$. 
venu, peur d'être trahi par ce fils dont il a fait son page et son plus sûr complice, peur de tomber dans un traquenard, peur des mauvaises nouvelles, une véritable " agonie " prompte à faire d'un mécréant le plus crédule des hommes ${ }^{48}$. Montherlant a publié en 1952 un recueil de textes sur la tauromachie qu'il a intitulé Le Plaisir et

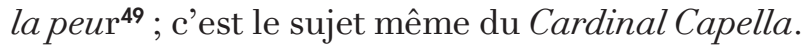

\section{Pierre DUROISIN}

480/8, chaussée de Wavre

B - 1040 Bruxelles

duroisin.lenglez@scarlet.be

48 “Quand il avait peur il croyait à n'importe quoi ». On a vu que l'abbé de Pradts des Garçons trouve ou retrouve la foi avant de mourir ; on aurait aimé savoir ce que Montherlant pensait faire de sa note « hostie après double sacrilège (il a bu) qui mit en lui une brûlure qui fut autre que celle de son âme », et dans quelles dispositions Capella serait mort, s'il devait mourir.

49 Tiré à 150 exemplaires seulement et sans nom de lieu ni d'éditeur. 